\title{
Glycosaminoglycan-Mimetic Signals Direct the Osteo/Chondrogenic Differentiation of Mesenchymal Stem Cells in a Three-Dimensional Peptide Nanofiber Extracellular Matrix Mimetic Environment
}

\author{
Elif Arslan, Mustafa O. Guler,* and Ayse B. Tekinay* \\ Institute of Materials Science and Nanotechnology, Nanotechnology Research Center (UNAM), Bilkent University, Ankara 06800, \\ Turkey
}

\section{Supporting Information}

\begin{abstract}
Recent efforts in bioactive scaffold development focus strongly on the elucidation of complex cellular responses through the use of synthetic systems. Designing synthetic extracellular matrix (ECM) materials must be based on understanding of cellular behaviors upon interaction with natural and artificial scaffolds. Hence, due to their ability to mimic both the biochemical and mechanical properties of the native tissue environment, supramolecular assemblies of bioactive peptide nanostructures are especially promising for development of bioactive ECM-mimetic scaffolds. In this study, we used glycosaminoglycan (GAG) mimetic peptide nanofiber gel as a three-dimensional (3D) platform to investigate how cell lineage commitment is altered by external factors. We observed that amount of fetal bovine serum (FBS) presented in the cell media had synergistic effects on the ability of GAGmimetic nanofiber gel to mediate the differentiation of mesenchymal stem cells into osteogenic and chondrogenic lineages. In particular, lower FBS concentration in the culture medium was observed to enhance osteogenic differentiation while higher amount FBS promotes chondrogenic differentiation in tandem with the effects of the GAG-mimetic 3D peptide nanofiber network, even in the absence of externally administered growth factors. We therefore demonstrate that mesenchymal stem cell differentiation can be specifically controlled by the combined influence of growth medium components and a 3D peptide nanofiber environment.
\end{abstract}

\section{INTRODUCTION}

Functional imitation of extracellular matrix (ECM) elements is a potent means of modulating cellular behavior for regenerative medicine. The composition of the natural ECM is tissuespecific and heterogeneous, but generally features large macromolecule assemblies that serve as three-dimensional (3D) scaffolds for the attachment of cells. ${ }^{1}$ Due to their versatility and relative ease of isolation and cultivation, stem cells are used in many biomedical applications and clinical therapies. $^{2}$ The precise control of stem cell differentiation pathways is therefore a major issue in regenerative medicine, as the heterogeneous and unpredictable nature of stem cells makes their use problematic in medical applications. ${ }^{3}$ Therefore, synthetic scaffolds are commonly used to provide an environment conductive for the regeneration of the tissue of interest by assisting in the survival of stem cells or directing their commitment and differentiation through bioactive signals. ${ }^{4}$ However, the $2 \mathrm{D}$ culturing of mesenchymal stem cells in particular is problematic for cell differentiation studies, as the lack of a 3D scaffold introduces an unnatural factor to the growth environment by preventing cells from fully making contact with the material. ${ }^{5}$ By contrast, mesenchymal stem cells in $3 \mathrm{D}$ cultures retain their stellate morphology and polarization in the antero-posterior axis, which supports their survival by hindering apoptosis. ${ }^{5}$ Therefore, the development of $3 \mathrm{D}$ microenvironments capable of mimicking the function of natural ECM components is critical for both regenerative medicine and basic molecular biology research. Considering their critical role in tissue remodeling, the inclusion of GAGmimetic molecules in a 3D network is an advantageous strategy for the priming of stem cells for osteogenic and chondrogenic differentiation. The GAG molecules provide mechanical and biochemical features, which should be integrated into synthetic scaffold materials to mimic their functions. ${ }^{4,6,7}$ Moreover, a variety of media components and culture conditions have been demonstrated to exhibit distinct effects on the differentiation of stem cells such as inclusion of growth factors, fetal bovine serum (FBS), etc. ${ }^{8,9}$

Here, we investigated the ability of 3D GAG-mimetic peptide nanofiber gels to mediate the differentiation of mesenchymal stem cells into two fundamental mesenchyme-originated lineages. Two distinct culture media containing different amounts of FBS were used to control external factors. The differentiation patterns of the cells were determined and

Received: December 5, 2015

Revised: February 2, 2016

Published: February 3, 2016 
possible cell-material interactions responsible for causing these patterns were analyzed to evaluate the modulatory effect of serum on the culturing of stem cells in the context of bioactive signals provided by a $3 \mathrm{D}$ peptide nanofiber network. The $3 \mathrm{D}$ GAG-mimetic peptide nanofiber gel niche was observed to demonstrate distinct effects on the osteogenesis and chondrogenesis of mesenchymal stem cells in low- and normal-serum environments, suggesting that serum presence can be used to directly alter the ability of a 3D scaffold to enhance stem cell differentiation toward osteogenic or chondrogenic lineage. Nonbioactive peptide nanofiber gels, however, did not exhibit this property, suggesting that the bioactivity of GAG-mimetic nanofibers was necessary for the induction of differentiation by the culture medium. Our straightforward and simple design demonstrates that the bioactivity of a 3D peptide nanofiber scaffold, in tandem with the effect of serum amount in the culture medium, is able to exhibit strong modulatory effects on the differentiation of stem cells without using any growth factor supplements.

\section{EXPERIMENTAL SECTION}

Materials. 9-Fluorenylmethoxycarbonyl (Fmoc) and tertbutoxycarbonyl (Boc) protected amino acids, [4-[ $\alpha$ - $\left(2^{\prime}, 4^{\prime}\right.$-dimethoxyphenyl) Fmoc aminomethyl]enoxy] acetamidonorleucyl-MBHA resin (Rink amide MBHA resin), Fmoc-Glu(OtBu)-Wang resin and 2(1H-benzotriazol-1-yl)-1,1,3,3-tetramethyluronium hexafluorophosphate (HBTU) were purchased from NovaBiochem and ABCR. Coverglasses and tissue culture plates (24-well) were purchased from Deckglaser and $\mathrm{BD}$. All other chemicals and materials used were analytical grade and obtained from Invitrogen, Fisher, Merck, Alfa Aesar, and Sigma-Aldrich. TGF $\beta$, differentiation media and collagen type I (C7661) were purchased from Sigma. Live/Dead Assay (L3224), Alamar Blue and other cell culture materials were purchased from Invitrogen. Antibodies were purchased from Abcam.

Synthesis and Characterization of Peptide Amphiphile Molecules. Fmoc solid-phase peptide synthesis method was performed to synthesize Lauryl-Val-Val-Ala-Gly-Glu-Asp-Lys-( $p$-sulfobenzoyl)-Ser-Am (GAG-PA), Lauryl-Val-Val-Ala-Gly-K-Am (K-PA), Lauryl-Val-Val-Ala-Gly-Glu-Arg-Gly-Asp (RGD-PA), and Lauryl-ValVal-Ala-Gly-E (E-PA). Rink amide MBHA resin (for GAG-PA and K$\mathrm{PA})$, Fmoc-Glu-(OtBu)-Wang resin (for E-PA) and Fmoc-Asp(OtBu)-Wang resin (for RGD-PA) were used as solid supports. All amino acid couplings were performed with 2 equiv of Fmoc-protected amino acid, 1.95 equiv of HBTU and 3 equiv of $N, N$-diisopropylethylamine (DIEA) in DMF for $3 \mathrm{~h}$. Twenty percent piperidine/ dimethylformamide (DMF) solution and $10 \%$ acetic anhydrideDMF solution were used for Fmoc deprotections for $20 \mathrm{~min}$ and acetylation of the unreacted amine groups after each coupling step, respectively. Lauric acid as the source of the terminal lauryl group was coupled similar to amino acids. For GAG-PA synthesis, sulfobenzoic acid was added to the lysine side chain; lysine residues with 4methytrityl (Mtt) side chain protection was used for the selective deprotection of amine groups. Mtt removal was performed by shaking resins for $5 \mathrm{~min}$ in TFA:TIS: $\mathrm{H}_{2} \mathrm{O}: \mathrm{DCM}$ in a ratio of 5:2.5:2.5:90. The cleavage of the peptides from the resin and deprotection of acid labile protected amino acids were carried out with a mixture of trifluoroacetic acid (TFA):triisoproplysilane (TIS):water in a ratio of 95:2.5:2.5 for $2.5 \mathrm{~h}$. Excess TFA was removed by rotary evaporation. Ice-cold diethyl ether was used to triturate the remaining residue and the resulting white pellet was freeze-dried. Peptide amphiphile molecules were identified and analyzed by reverse phase HPLC on an Agilent 6530 accurate-Mass Q-TOF LC/MS equipped with an Agilent 1200 HPLC. The K-PA molecule was purified on an Agilent 1200 HPLC system by using a Zorbax prepHT 300CB-C8 column with a water-acetonitrile (0.1\% TFA) gradient. GAG-PA, E-PA, and RGD-PA have acidic characters, so Agilent Zorbax Extend-C18 $(50 \times$ $2.1 \mathrm{~mm})$ column with a water/acetonitrile mixture $\left(0.1 \% \mathrm{NH}_{4} \mathrm{OH}\right)$ was used. Their purification was performed on a Zorbax Extend C18 prep-HT with a water/acetonitrile $\left(0.1 \% \mathrm{NH}_{4} \mathrm{OH}\right)$ gradient.

Preparation and Characterization of the Self-Assembled Peptide Nanofiber Gels for 3D Cell Culture Experiments. Negatively charged PA solutions (GAG-PA, E-PA, RGD-PA) were prepared in the cell culture medium (Dulbecco's modified Eagle medium (DMEM) + 10\% FBS), while positively charged PA solutions were prepared in $0.25 \mathrm{M}$ sucrose solution. For nanofiber formation, Lauryl-VVAGEGD-K(p-sulfobenzoyl)-S-Am (GAG-PA), Lauryl-VVAGERGD (RGD-PA) and Lauryl-VVAGK-Am (K-PA) were mixed at a 3:1:5 mM ratio in order to form a GAG-mimetic peptide gel. In a similar manner, Lauryl-VVAGE (E-PA), Lauryl-VVAGERGD (RGDPA) and Lauryl-VVAGK-Am (K-PA) were mixed at a 3:1:5 mM ratio for nonbioactive gel formation. Mesenchymal stem cell pellets were suspended in RGD-PA solution (in culture medium) and mixed inside wells alongside either GAG-PA or E-PA with K-PA to produce threedimensional GAG-nanofiber (composed of GAG-PA/RGD-PA/K-PA) and E-nanofiber (composed of E-PA/RGD-PA/K-PA) scaffolds. Three $\mathrm{mL}$ of cell medium was added to the wells and the gels were incubated for $30 \mathrm{~min}$ at $37^{\circ} \mathrm{C}$. Collagen I (Life Technologies) gel was used as control.

For scanning electron microscopy (SEM) imaging, 3D peptide gels with cells were washed with PBS and fixed with $2 \%$ glutaraldehyde/ PBS for $2 \mathrm{~h}$. Peptide gels without cells were also prepared at identical concentrations to analyze the changes in the structural features of gels in the presence of cells. Following three washing steps with PBS, samples were dehydrated in graded ethanol solutions, starting with $20 \%$ ethanol and proceeding to absolute ethanol for $10 \mathrm{~min}$ at each step. Samples were dried with a Tourismis Autosamdri-815B critical point drier, coated with $10 \mathrm{~nm} \mathrm{Au} / \mathrm{Pd}$ and imaged with a FEI Quanta 200 FEG SEM.

Transmission electron microscopy (TEM) analysis was performed with FEI Tecnai G2 F30 TEM at $200 \mathrm{kV}$. High-angle annular dark field detector was used for images taken in STEM mode. Seven $\mathrm{mM}$ of GAG and E-PA gel nanofiber systems were first diluted fifty-fold and then dropped on a 200-mesh copper TEM grid. Samples were left at room temperature for $7 \mathrm{~min}$, stained with $2 \mathrm{wt} \%$ uranyl acetate for another 2 min and air-dried prior to TEM imaging.

Circular dichroism $(\mathrm{CD})$ measurements were performed to analyze the secondary structures of PA nanofibers. Jasco J-815 CD spectrophotometer was used for $\mathrm{CD}$ analysis. All samples were measured at hundred-fold dilutions of concentrations used in cell culture experiments. For each measurement, $300 \mu \mathrm{L}$ of PA material was transferred into a $1 \mathrm{~mm}$ quartz cuvette, which was inverted gently to mix the sample without damaging the assembled nanostructures. Spectra were obtained at room temperature at a range of 300 to 190 $\mathrm{nm}$, with a data interval of $1 \mathrm{~nm}$ and a scanning speed of $100 \mathrm{~nm} / \mathrm{min}$. The results were expressed as mean residue ellipticity and converted to the unit of deg $\cdot \mathrm{cm}^{2} \cdot \mathrm{dmol}^{-1}$.

Oscillatory rheology measurements were performed with an Anton Paar Physica MCR301 system. A $25 \mathrm{~mm}$ parallel plate with a gap distance of $0.5 \mathrm{~mm}$ was used at $25^{\circ} \mathrm{C}$ for all measurements. The total gel volume was adjusted as $160 \mu \mathrm{L}$. PA solutions for 3D cell cultures were freshly prepared as $10 \mathrm{mM}$ K-PA, $6 \mathrm{mM}$ GAG-PA, $6 \mathrm{mM}$ E-PA, and $2 \mathrm{mM}$ RGD-PA, and then sonicated for $30 \mathrm{~min}$. Gels were prepared using the GAG-RGD-K-PA and E-RGD-PA combinations. The negatively charged PA (GAG-PA or E-PA) was first loaded at the center of the stage and mixed with RGD-PA; the positively charged KPA was then mixed with the sample in a spiral pattern. The upper plate was adjusted to the $0.5 \mathrm{~mm}$ position and the gel was incubated in this position for $15 \mathrm{~min}$ prior to measurement. For strain sweep measurements, angular frequency was kept constant at $10 \mathrm{rad} / \mathrm{s}$, and strain was increased between 0.1 and $100 \%$. Storage and loss moduli were recorded at each strain value. All rheology measurements were performed on gels with and without cells with three replicates.

Zeta potential measurements were performed for the analysis of the surface charge of the PA molecules and PA nanofibers, which are 20 times diluted from the gel concentrations used for the in vitro experiments. The PA solutions were prepared in $\mathrm{ddH}_{2} \mathrm{O}$. Measurements were performed with Malvern Nano-ZS zetasizer and 

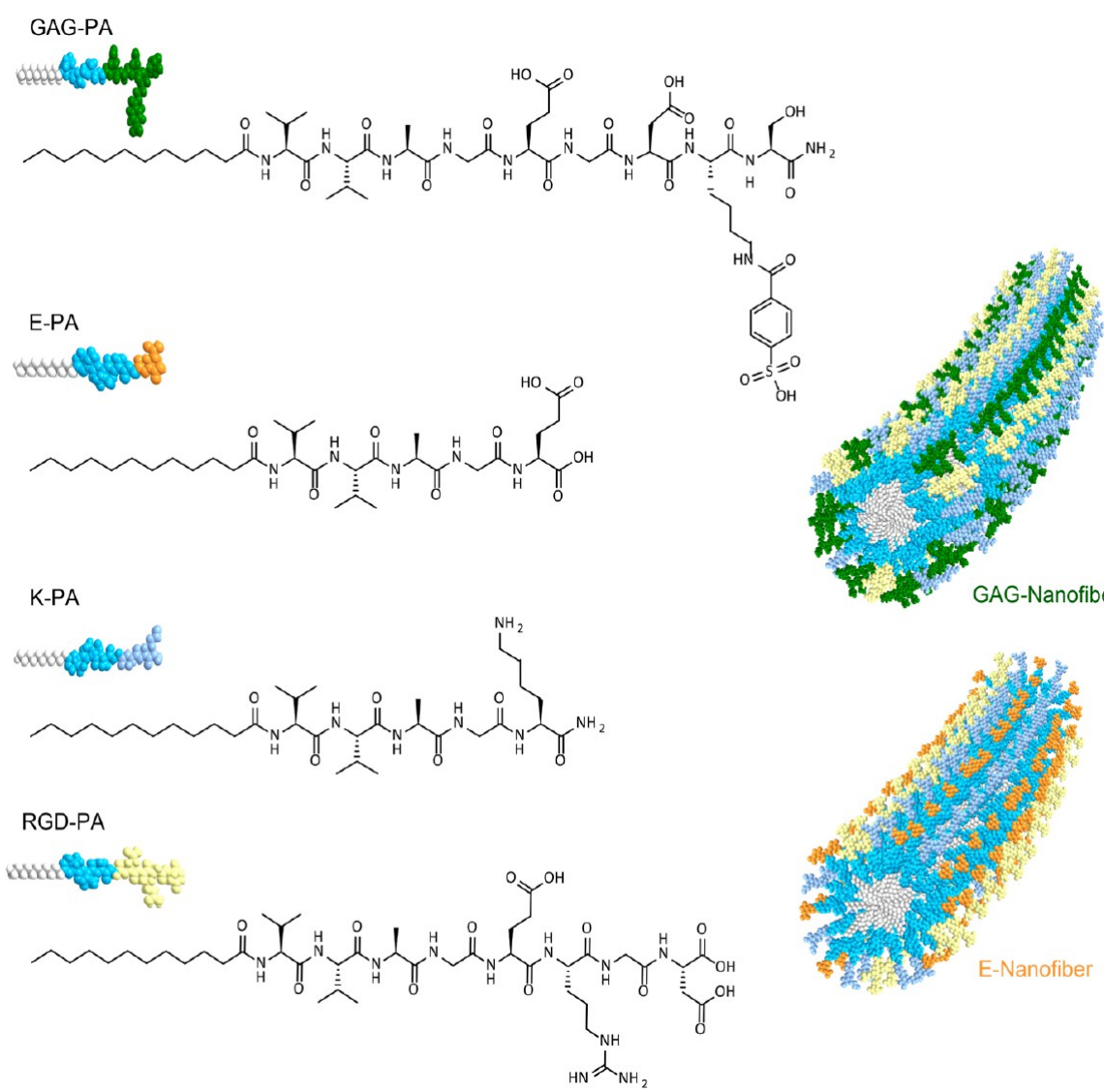

Figure 1. Chemical Structures of peptide amphiphile molecules. GAG-PA: Lauryl-VVAGEGD-K(p-sulfobenzoyl)-S-Am; E-PA: lauryl-VVAGE, KPA: Lauryl-VVAGK-Am; RGD-PA: Lauryl-VVAGERGD.

Smoluchovski approach was used to calculate the Zeta Potential values.

3D Cell Culture and Maintenance. The rMSCs (rat mesenchymal stem cells, Invitrogen S1601-100) at passage 8 were used in all 3D cell culture experiments, including viability, rheology, GAG deposition and differentiation assays. The rMSCs were initially cultured in DMEM supplemented with 10\% FBS (Maintenance Media) in tissue culture plates at standard culture conditions (at $37^{\circ} \mathrm{C}$ under $5 \% \mathrm{CO}_{2}$ ). After 1 day of incubation, maintenance medium was replaced with maintenance medium (MT) or DMEM supplemented with $2 \%$ FBS (low FBS medium). Maintenance medium supplemented with $10 \mathrm{mM} \beta$-glycerophosphate, $0.2 \mathrm{mM}$ ascorbic acid, and $100 \mathrm{nM}$ dexamethasone was used for osteogenic differentiation on tissue culture plate (TCP).

Viability Analyses. 3D cell cultures were prepared in 24 well plates in MT medium, and cell viability was analyzed by Alamar Blue (Invitrogen) and Live/Dead (Life Technologies) assays. Five $\times 10^{5}$ rMSCs were seeded into gels and their viability was measured at days 3,7 , and 14 by measuring the fluorescence and absorbance of the reagent by spectrophotometry for the Alamar Blue assay, and imaging the cells by fluorescence microscopy and confocal microscopy for the Live/Dead Assay.

Sulfated Glycosaminoglycan Deposition Analysis. Quantification of sulfated glycosaminoglycans was done by dimethylmethylene blue assay. Cell cultures were digested in papain digestion buffer (100 $\mathrm{mM}$ sodium phosphate buffer/10 $\mathrm{mM} \mathrm{Na}{ }_{2} \mathrm{EDTA} / 10 \mathrm{mM}$ L-cysteine/ $0.125 \mathrm{mg} / \mathrm{mL}$ papain) overnight at $65^{\circ} \mathrm{C}$, and total DNA amount per gel was measured with Qubit dsDNA quantitation kit (Invitrogen) to normalize the sulfated glycosaminoglycan content. To produce standard curves, diluted chondroitin sulfate standards (from 0 to 35 $\mu \mathrm{g} \mathrm{mL}^{-1}$ ) were prepared for dimethyl methylene blue (DMMB) assay. One hundred microliters of DMMB solution (16 mg L-11,9dimethylmethylene blue, $40 \mathrm{mM}$ glycine, $40 \mathrm{mM} \mathrm{NaCl}, 9.5 \mathrm{mM}$
$\mathrm{HCl}, \mathrm{pH}$ 3.0) was added to $40 \mu \mathrm{L}$ of papain-digested solutions and to standard samples. Then, optical densities (ODs) of the solutions were measured at $525 \mathrm{~nm}$ wavelength on a microplate reader. The absorbance of the control groups (gels without cells) was subtracted from the absorbance values of the experimental groups.

Gene and Protein Expression Analysis. Gene expression analyses were performed by amplifying markers for chondrogenesis (Sox9 and collagen II) and osteogenenesis (collagen I and Runx2) by quantitative RT-PCR (qRT-PCR). Total RNAs of rMSCs in 3D PA gels were isolated using TRIzol (Invitrogen) according to the manufacturer's instructions. Yields and purities of extracted RNA were assessed by Nanodrop 2000 (Thermoscientific). Primer sequences were designed using Primer 3 software (Table S2). cDNA synthesis from RNA and qRT-PCR were performed using SuperScript III Platinum SYBR Green One-Step qRT-PCR Kit according to the manufacturer's protocol. Reaction conditions were briefly as follows: $55^{\circ} \mathrm{C}$ for $5 \mathrm{~min}, 95^{\circ} \mathrm{C}$ for $5 \mathrm{~min}, 40$ cycles of $95^{\circ} \mathrm{C}$ for $15 \mathrm{~s}, 60^{\circ} \mathrm{C}$ for $30 \mathrm{~s}$, and $40{ }^{\circ} \mathrm{C}$ for $1 \mathrm{~min}$, followed by a melting curve to confirm product specificity. Reaction efficiencies were evaluated for each primer set through standard curves using 5-fold serial dilutions of total RNA. For the analysis of expression, primary gene expression data were normalized by the expression level of GAPDH. A comparative $\mathrm{Ct}$ method was used to analyze the results.

Flow cytometry was performed to better quantify the differentiation potential of rMSCs in PA gels; expression of Sox9 and Runx2 proteins were analyzed to evaluate whether cells within gels have committed into osteogenic or chondrogenic lineages. Prior to flow cytometry analysis, the gel construct was disintegrated by collagenase/trypsin treatment and a cell strainer (Falcon) was used to isolate cells from gels. Approximate yield of cell filtration was calculated with cell counting formulation after staining with Trypan Blue, and we found that $95-100 \%$ of cells were successfully filtered and isolated for subsequent analyses. The supernatant was then collected and 
A

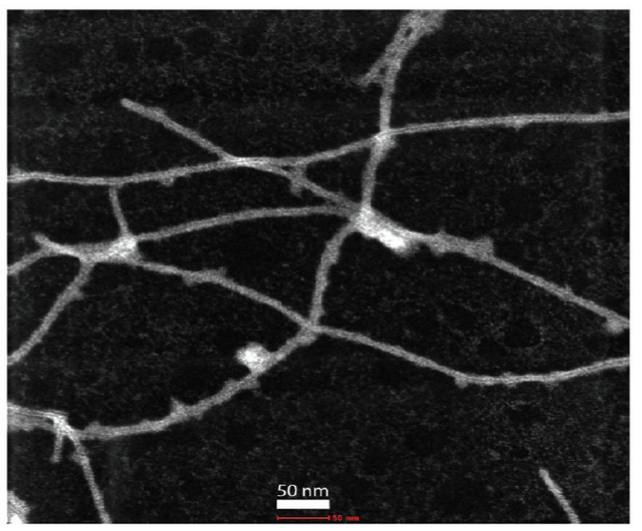

B

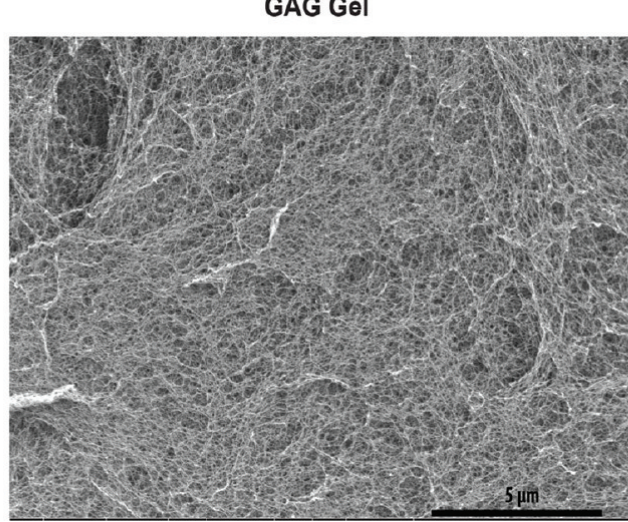

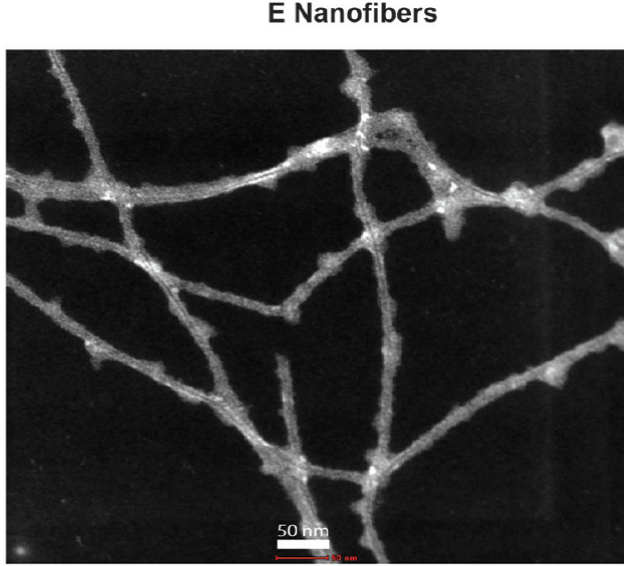

E Gel

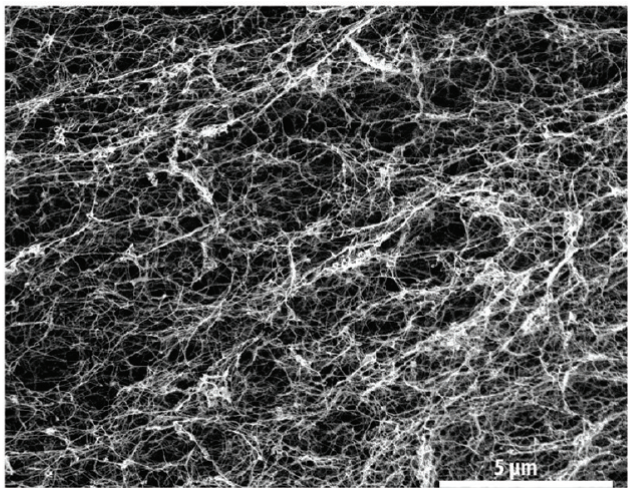

C
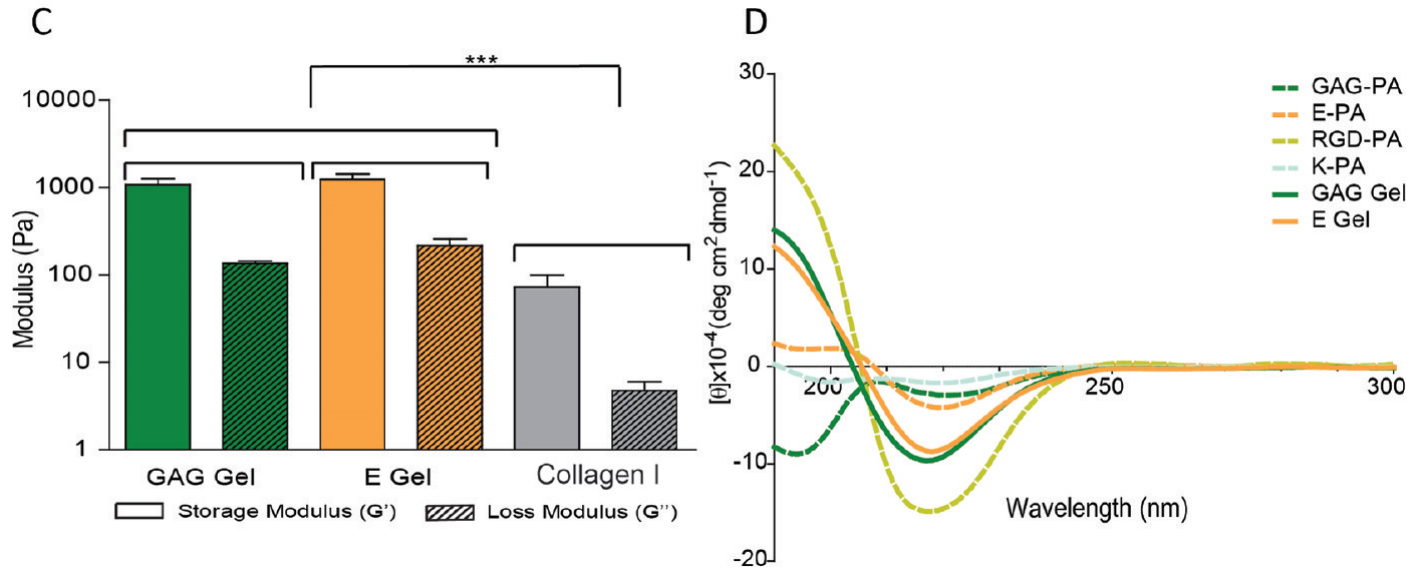

Figure 2. Characterization of peptide amphiphile molecules and peptide nanofiber gels. (A) STEM images of GAG and E nanofibers, scale bar $=50$ $\mathrm{nm}$. (B) SEM images of GAG- and E-gels, scale bar $=5 \mu \mathrm{m}$. (C) Rheological analyses of peptide nanofiber gels and collagen I gel, $* * * p<0.001$. Equilibrium moduli of the collagen I gel are significantly less than the equilibrium moduli of peptide nanofiber gels. (D) Circular dichroism spectra of peptide amphiphile solutions and gels.

centrifuged at $2500 \mathrm{rpm}$ for $5 \mathrm{~min}$. The cell pellet was washed twice with PBS, resuspended, and fixed with $4 \%$ paraformaldehyde for 15 $\mathrm{min}$ at room temperature. The solution was then centrifuged at 2500 rpm for $5 \mathrm{~min}$, and the cell pellet was resuspended and permeabilized in $0.2 \%$ Tween-20/PBS for $15 \mathrm{~min}$ at room temperature. Primary antibody solutions of either Sox9 [Anti-Sox9 antibody, (ab185230)] or Runx2 [Anti-Runx2 antibody (ab76956)] or their corresponding isotypes, rabbit monoclonal IgG (ab172730) and mouse monoclonal IgG2a (ab170191), were prepared in 3\% BSA/PBS solution at concentrations recommended by the manufacturer (Abcam) and used to stain the cells for $1 \mathrm{~h}$. After primary antibody staining, cells were washed with permeabilizing solution, pelleted by centrifugation and stained with secondary antibodies [Goat Anti-Mouse IgG H\&L (Alexa
Fluor 488) (ab150113) for Runx2 and Goat Anti-Rabbit IgG H\&L (Cy5 ) preadsorbed (ab97077) for Sox9] for $1 \mathrm{~h}$. After washing twice with permeabilizing agent, the cells were again pelleted by centrifugation and resuspended in PBS prior to cytometry analysis. The rMSCs grown in corresponding differentiation media (either osteogenic or chondrogenic media) were used as positive controls. BD AccuriTM C6 flow cytometer system was used for cytometry, and at least 50000 events were analyzed for the measurements. For data analysis, BD AccuriTM C6 software was used according to the manufacturer's instructions.

Statistical Analysis. All data are presented as mean \pm SEM (standard error of mean). The significance of differences between groups was determined with either one-way or two-way analysis of 
A

GAG Gel Niche

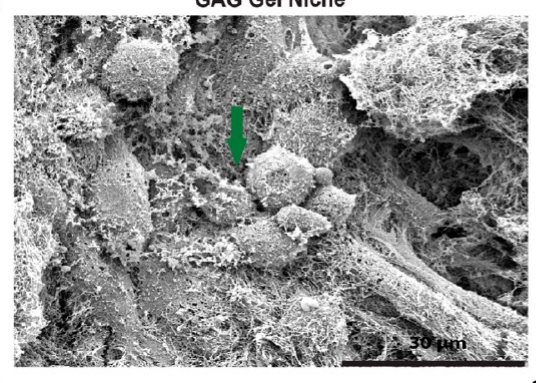

B

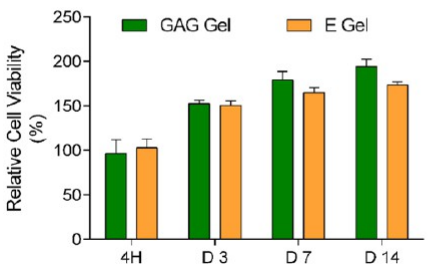

D
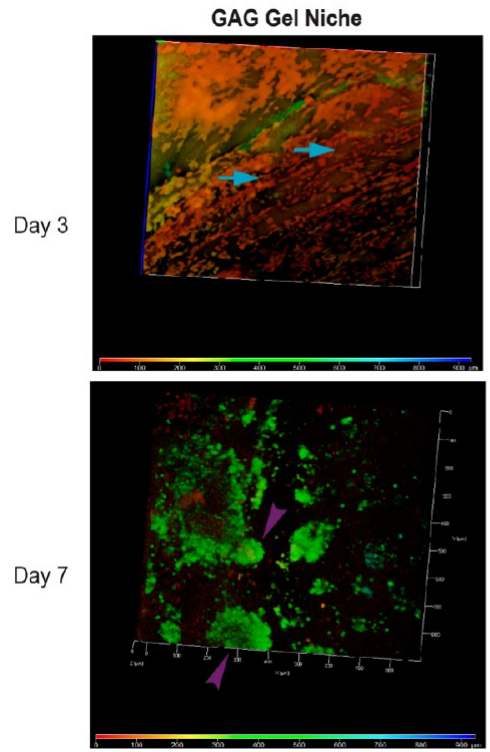

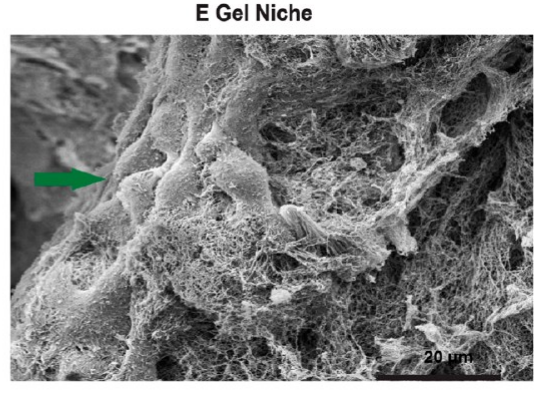

C

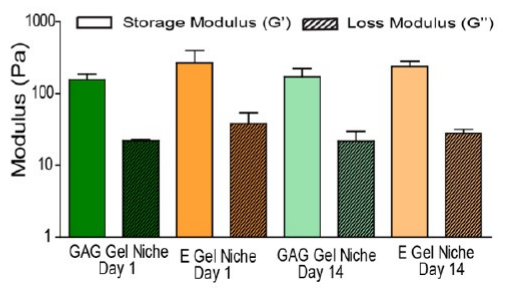

E Gel Niche
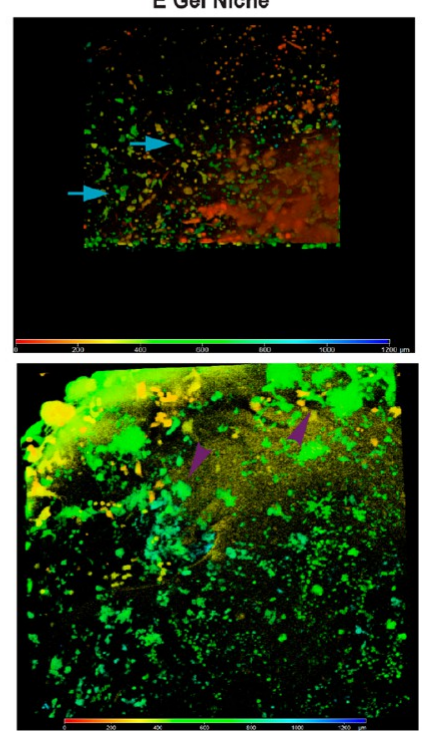

Figure 3. Structural, morphological and mechanical properties of the peptide nanofiber gel niches. (A) SEM images of cells cultured within GAGand E-gel niches at day 3, green arrows show the cell clusters interacting with peptide nanofibers in gel niche, scale bar $=30 \mu \mathrm{m}$ for GAG-gel and scale bar $=20 \mu \mathrm{m}$ for E-gel. (B) Analysis of relative cell viability of rMSCs within PA gel niche for 14 days. (C) Rheology analyses of peptide nanofiber gel niches incubated with cells for 1 day and 14 days. (D) Confocal microscopy analyses of cellular localization within peptide nanofiber gels following 3 and 7 days of culture. Blue and purple arrows show the cells and cell clusters within gel niche. Colored-scale bar shows the depth of gel niches; the corresponding cellular localizations are shown in depth-dependent colors (using a red to blue scale for surface- to bottom-depth).

variance (ANOVA) with posttests (Tukey's/Bonferroni). Differences were considered significant at $p<0.05$, except where noted.

\section{RESULTS AND DISCUSSION}

Design, Synthesis and Characterization of the SelfAssembled PA Molecules and 3D PA Nanofiber Gels. The 3D gels provide a native-like microenvironment for the growth and differentiation of cells, thereby filling the gap between in vitro scaffold systems and in vivo tissue architectures. In this work, we studied the potential of a glycosaminoglycan-mimetic peptide nanofiber system for the induction of mesenchymal stem cell differentiation into osteo/ chondrogenic lineages. Peptide nanofiber gels resemble the native tissue in having a nanofibrous structure akin to the collagenous fiber network found in the extracellular matrix. In addition to sharing a fibrous nature, both gel and ECM environments are also rich in water and present functional groups such as sulfonate, carboxylate and hydroxyl moieties (Figure 1).

Two gels were used for the present study: GAG-gel (GAGPA/RGD-PA/K-PA) contains bioactive residues, while the control E-gel (E-PA/RGD-PA/K-PA) was used as the experimental control (Table S1). GAG-gel was decorated with sulfonate, hydroxyl and carboxylate groups to imitate the structure of heparan sulfate glycosaminoglycans, while the E-gel presented carboxylate group and lacked sulfonate and hydroxyl groups (Figure 1). Both gels were formed by supramolecular interactions between peptide amphiphile molecules, which produce $\beta$-sheet containing nanofibers through self-assembly. This process is driven largely by charge neutralization between oppositely charged peptide amphiphiles, and further influenced by cohesive forces such as hydrogen bonding and van der 
Waals, hydrophobic, and electrostatic interactions. ${ }^{4,10,11}$ In this study, both gels contain RGD-PA, a bioactive sequence that enhances the adhesion of cells to the scaffold through integrinmediated interactions between nanofiber surfaces and the cell membrane. The RGD sequence was chosen as a wellestablished epitope for cell adhesion, ${ }^{12,13}$ and in our optimization studies, we observed that the presence of the RGD sequence (at a 1:7 RGD-PA:gel ratio) increases the attachment and survival of cells in peptide nanofiber gels.

Peptide amphiphile molecules were characterized by liquid chromatography-mass spectrometry (LC-MS) and purified by preparative HPLC prior to use (Figure S1). STEM (scanning transmission electron microscopy) of negatively stained coassembled peptide nanofibers (Figure 2A) revealed that GAG and $E$ nanofiber networks were very similar to each other and that the peptide fibers comprising the gels had high aspect ratios and nanometer-scale diameters $(10-20 \mathrm{~nm})$. The sizes of these nanofibers are similar to the fibrous collagen and glycoproteins found in the ECMs of both cartilage and bone. $^{14,15}$ SEM images of gels (Figure 2B) demonstrated the formation of a porous nanofiber network following the coassembly of PA molecules. The secondary structures of both gels were also analyzed by $\mathrm{CD}$ (Figure $2 \mathrm{D}$ ). CD spectra of both GAG- and E-gels were found to correspond to the $\beta$-sheet conformation, with a chiral absorbance at $\sim 220 \mathrm{~nm}$ (Figure $2 \mathrm{D})$. In addition, pure GAG-PA, E-PA and K-PA molecules were not observed to form $\beta$-sheets at analyzed concentration. However, the CD spectrum of pure RGD-PA was found to show distinctive $\beta$-sheet features, which can be attributed to the self-neutralization of this peptide due to the decreased electrostatic repulsion between peptide amphiphile molecules in the cell medium. As the gels used for cell culture experiments were higher in concentration (up to $7 \mathrm{mM}=\sim 1 \% \mathrm{w} / \mathrm{v}$ ) compared to these in $\mathrm{CD}$ measurements, $\beta$-sheet formation is expected to be prominent in all gel scaffolds used. SEM images also suggest that the peptide nanofibers form a mesh-like structure that is similar to the fibrous environment of the extracellular matrix and therefore conductive to the attachment of cells.

Oscillatory rheology analyses were performed to assess the mechanical properties of peptide nanofiber gels. Time sweep, frequency sweep, and strain sweep rheology analyses were conducted in order to investigate the gel formation mechanics and viscoelastic properties of the scaffold material (Figure 2C and Figure S2-S3). Storage moduli of both GAG- and E-gels are found to be higher than their loss moduli at their cell culture concentrations, suggesting that the materials are gels in the strict sense. In addition, mechanical analyses were performed for gel niches containing encapsulated cells, and gels were observed to experience a slight decrease in stiffness moduli after 1 and 14 days of cell culture (Figure 3C). This can be attributed to their degradation by cellular enzymes and partial replacement with the native extracellular depositions of cells. In addition, $5 \times 10^{5}$ rMSCs were encapsulated in each gel, which introduces a considerable void in the gel upon encapsulation, which could result in decrease in the viscoelastic moduli of gels. Moreover, cells change the mechanical properties of gels during their physical reorganization through differentiation process and secrete their own ECM. However, this does not affect the integrity of gels, so gels provide 3D microenvironment to the high amount of cells for growth and proliferation for 2 weeks. Collagen I gel, a scaffold commonly used in bone and cartilage regeneration studies, was also used as a control $3 \mathrm{D}$ gel environment for comparison. ${ }^{16}$ Despite their mechanical properties to be used as scaffold in cell culture studies, collagen gels suffer from limitations such as immunogenicity, batch-to-batch variances and side effects resulting from viral and bacterial contamination, which are inherent to animal-derived scaffolds and necessitate the use of artificial matrices for the safe and efficient recovery of bone and cartilage injuries. ${ }^{17}$ Equilibrium modulus values of peptide nanofiber gels and collagen I gel suggests that both GAG- and E-gels have similar moduli, while the modulus values associated with collagen I gel are significantly less than peptide gels. The higher storage moduli of peptide gels could be attributed to concentration differences, as higher polymer concentrations result in higher stiffness moduli. Differences in polymerization conditions, cross-linking degrees and molecular weights of peptide amphiphile molecules and collagen 1 also contribute to the discrepancy in the viscoelastic properties of their gels. ${ }^{18-20}$ However, both peptide nanofiber gels are at the same concentration, display similar morphologies, and exhibit comparable viscoelastic properties. As such, the possible bioactivity of the materials should be attributed to biochemical signatures presented in peptide gels rather than mechanical differences. Overall, rheological analysis revealed that the peptide amphiphile nanofibers displayed elastic solid-like behavior with high water content, which is consistent with the interwoven matrices of both structures observed under electron microscopy imaging. In addition, the mechanical stability of the gels was strong enough to support cell growth in both peptide gels, which were found to exhibit similar elastic properties.

While optimizing the gel concentrations, the combinations and concentrations of gels were selected as theoretically suitable to each other for proper comparison. We analyzed charges of the nanofibers and PA molecules dissolved in water at around $\mathrm{pH} 7$ with Zeta Sizer (Figure S11). Zeta potential measurements show both surface charge and stability of PA molecules and peptide nanofibers. When zeta potentials of molecules are high positive or high negative, there are repulsions between molecules, which prevents their selfassembly. When zeta potentials of molecules are between -10 and $+10 \mathrm{mV}$, these molecules are considered approximately neutral. However, when zeta potentials are less than $-30 \mathrm{mV}$ or greater than $+30 \mathrm{mV}$, they are expected to have strong anionic or strong cationic behavior, respectively. ${ }^{10,21}$ According to our results, the negative PA molecules (GAG-PA, E-PA, and RGD-PA) displayed strong negative potential, whereas, positive PA molecule (K-PA) displayed strong positive zeta potential in water. For peptide nanofibers, zeta potentials of both nanofibers were measured positive but not as strong as the soluble PA molecules. Therefore, the bioactivity of GAGPA nanofiber gel is attributed to specific epitopes presented on the nanofibers, which modulates the cellular behavior.

SEM images of gel niches (Figure 3A) were acquired to evaluate the distribution and morphology of cells embedded within gels. Attachment of the cells and cell clusters were demonstrated by SEM images. The nanofiber network was observed to resemble native cartilage and bone ECMs, potentially providing mechanical support and instructive cues for cells. It should be noted that while $3 \mathrm{D}$ microenvironments provide native-like material architectures for cells, they are often hindered by the insufficient transport of nutrients and waste products in and outside the scaffold. ${ }^{22}$ In our design, however, the porous structure of gel niches facilitates nutrient 
A

GAG-MT $\square$ GAG-LOW FBS $\square$ E-MT $\square$ E-LOW FBS

Sox 9
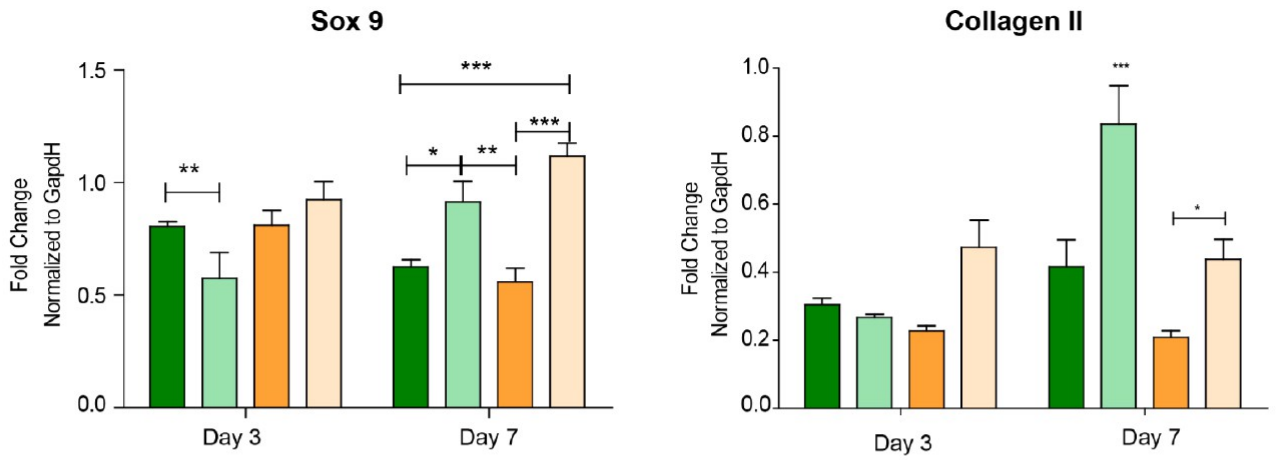

B

Runx 2
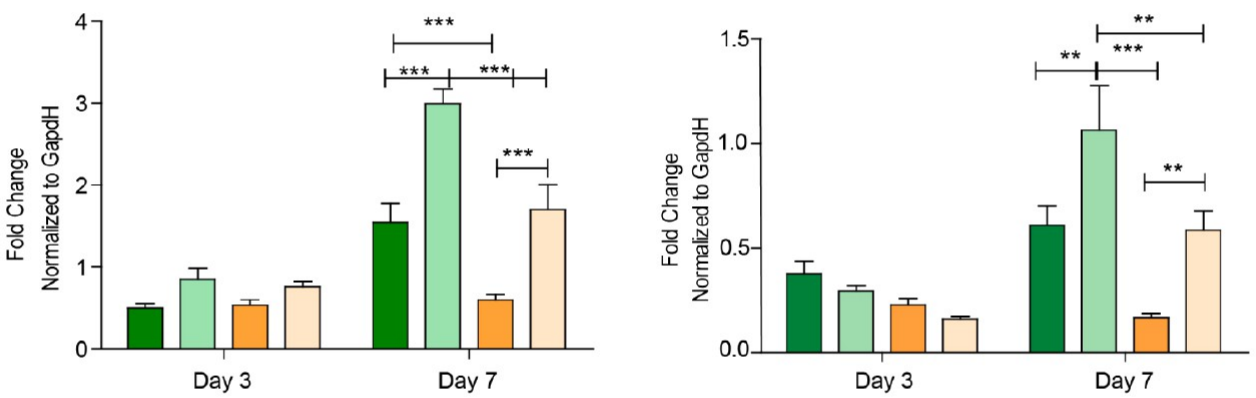

C

MT Media

Low FBS Media
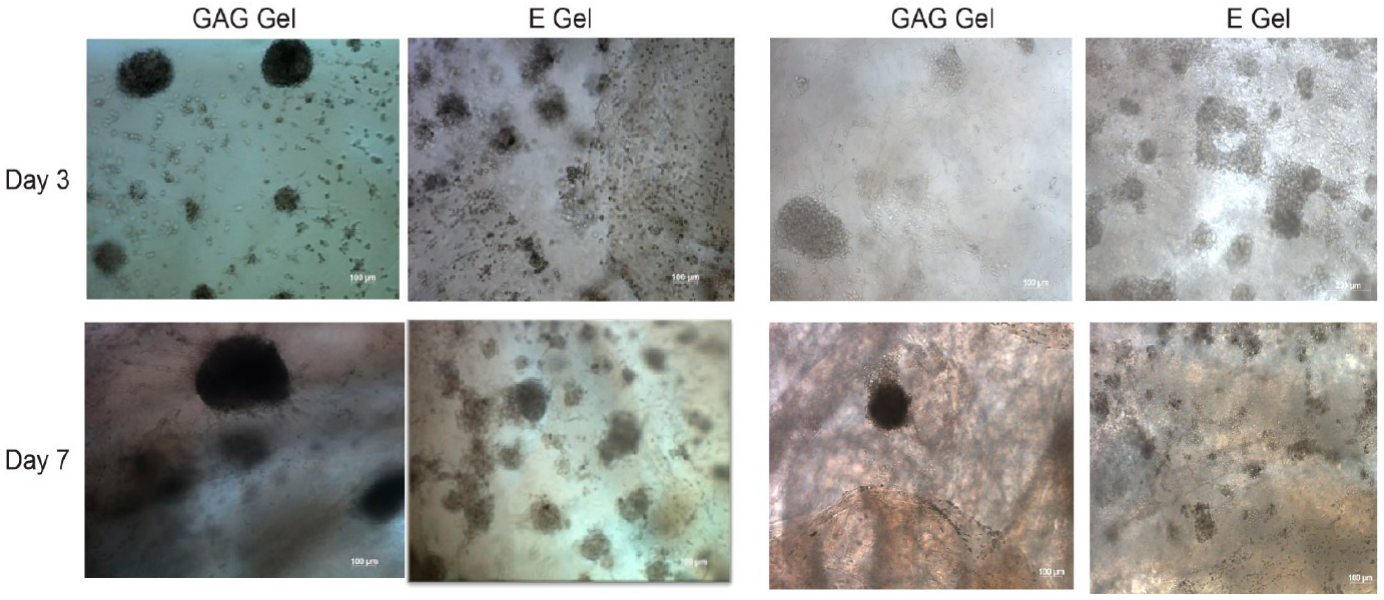

Figure 4. Differentiation analyses of rat mesenchymal stem cells embedded in GAG- and E-gel niches for 3 and 7 days. (A) Chondrogenic differentiation analyses by qRT-PCR. Expressions of Sox 9 and collagen II were quantified on days 3 and 7. (B) Osteogenic differentiation analyses by qRT-PCR. Expressions of Runx2 and collagen I were quantified on days 3 and 7. (C) Morphological tracking of cells in PA gel niches for 7 days, scale bar $=100 \mu \mathrm{m}$. (MT Media: 10\% FBS containing growth media, Low FBS Media: 2\% FBS containing media).

and waste transport, as well as allowing cell migration to occur for cellular reorganization during differentiation (Figure 2B and Figure 3A). Cells in gels displayed spherical morphologies similar to their in vivo condition, rather than the flattened shapes observed in $2 \mathrm{D}$ studies. ${ }^{22}$ As such, 3D peptide nanofiber gels appear to exhibit an ECM-like structure that is suitable for supporting the attachment, viability and growth of cells.

3D Peptide Gels Provide a Biocompatible Environment for Mesenchymal Stem Cells. In vitro cell culture analysis of gels is vital for assessing their ability to induce the differentiation of stem cells toward a specific lineage. Cell- materials interactions between mesenchymal stem cells and GAG-mimetic and control gels were evaluated by optical, SEM and confocal microscopy. According to results of Alamar Blue assay, both GAG- and E-gels are biocompatible and support the growth and proliferation of cells (Figure 3B). Live/Dead assay also showed that both living and dead cells were present in the scaffolds during 7 days of culture, and that the cells were distributed relatively uniformly within the gels (Figure 3D and Figure S4). It is noteworthy that the 3D microenvironment of gels is much closer to the native tissue than $2 \mathrm{D}$ cell culture, which further enhances the survival of stem cells. In particular, 
the highly porous structure of 3D scaffolds provides larger surface areas for cell attachment and proliferation, which enables the use of higher amount of cells in clinical applications. ${ }^{23}$ However, in $2 \mathrm{D}$ control, most of the cells were dead at day 7 because of over confluency (Figure S4). Confocal analysis revealed that cells could migrate across the depth of the gel, and by day 7 exhibited a tendency to form clusters (Figure 3D). The fact that extensive migration is possible within the $3 \mathrm{D}$ matrix suggests that cells may utilize proteolytic processes to locally modify matrix integrity. ${ }^{23}$ As such, our system is suitable for facilitating the dynamic turnover of the extracellular matrix, which is necessary for the migration and remodeling of differentiating cells.

Self-assembly is a major feature of supramolecular peptide amphiphile molecules and presents several opportunities in tissue engineering. The main advantage of the self-assembling systems is their adaptive nature, as their primary sequence can be modified to a great extent without substantially altering the gel's mechanical properties. In addition they provide a biocompatible, nanosized, porous, bioactive, and biodegradable gel environment that is well-suited for tissue engineering applications. ${ }^{4,24}$ In contrast, other gels used in regenerative medicine may suffer from drawbacks such as the lack of proper cell adhesion, insufficient bioactive signal delivery, nonbiodegradability, constrained structural stability (e.g., for gas foaming), residual porogen problems (for solvent casting), and complex and high cost processing (for phase separation and photolithography). ${ }^{25-31}$ Self-assembled peptide systems therefore provide considerable advantages as artificial 3D scaffolds for tissue regeneration. In addition, peptide nanofiber gels can be used as injectable gels to deliver cells into tissue defects, directly creating a cell/gel construct at the site of injury and allowing a more immediate tissue regeneration process compared to acellular scaffolds. ${ }^{32}$ Both bone and cartilage tissue defects are especially suitable for injectable cell/gel systems.

Stem Cell Differentiation Can Be Directed into Chondrogenic and Osteogenic Lineages in 3D Peptide Gels. The rMSCs were cultured on GAG-mimetic and nonmimetic gels for 7 days, and their differentiation profiles were evaluated through the analysis of their morphology, gene expression levels, glycosaminoglycan, and protein production by optical microscopy, qRT-PCR, DMMB, and flow cytometry. The influence of GAG-PA nanofibers in a $3 \mathrm{D}$ microenvironment was tested in tandem with different culture conditions to understand the processes underlying the lineage commitment of stem cells. Optical microscopy images of experimental groups suggest that mesenchymal stem cells in both GAG-PA and E-PA gels formed nodules, resulting in increased cell-to-cell interactions and cellular condensation (Figure 4C). Cells embedded in the GAG-gel niche formed larger nodules when compared to E-gel in the presence of both types of culture media. For both cartilage and bone differentiation, nodule formation is an important morphological change exhibited by stem cells, and is typically followed by mesenchymal condensation for the prefiguration of tissues. Therefore, morphology analysis suggests that stem cells in $3 \mathrm{D}$ gel environments follow the initial steps of osteo/chondrogenic differentiation. Upon this finding, differentiation capacities of mesenchymal stem cells at early (day 3 ) and middle/late (day 7) time periods were investigated in greater detail.

Cartilage differentiation was evaluated by the expression analysis of two cartilage specific markers, Sox9 (early) and collagen II (middle/late), by qRT-PCR. A significant difference was observed in the Sox9 mRNA expression in GAG- and E-gel niches in maintenance medium and low-FBS medium (Figure 4A): In the GAG-gel niche, the presence of low-FBS medium was found to downregulate Sox9 expression at day 3, although this trend was reversed by day 7 . In the E-gel niche, however, low-FBS medium increased Sox 9 expression at both days tested. Collagen II mRNA exhibited a trend similar to Sox9, with a transient downregulation at day 3 followed by upregulation at day 7. Low-FBS media was observed to induce collagen II expression, but the level of fold changes exceeded 1 for only the E-low FBS group. In addition to the analysis of mRNA expressions, sulfated glycosaminoglycan production patterns were also investigated by using DMMB assay (Figure S5). The pattern of sulfated GAG production at day 7 was found to correlate with Sox9 mRNA expression levels.

Two markers, Runx2 (early) and Collagen I (middle/late), were used for the evaluation of osteogenic differentiation (Figure 4B). The pattern of expressions at day 7 was similar for both osteogenic markers, while the fold changes for Runx2 expression were relatively higher compared to fold changes for Sox 9 expression. At day 3, no significant changes were observed for both markers across the groups. At day 7, however, expression levels of both osteogenic markers increased considerably in the GAG-gel group, suggesting that the combination of a GAG-like nanofiber scaffold and culture conditions had altered the differentiation pattern of the stem cells. This effect was especially pronounced in low FBS media. Indeed, the presence of a GAG-like nanofiber-containing culture environment and low levels of FBS can synergistically enhance the osteogenic differentiation of stem cells. Both culture conditions are also able to increase osteogenic differentiation to a lesser extent by themselves, as the GAGgel group exhibited higher expressions of Runx2 and collagen I compared to the nonbioactive scaffold, while cells in low-FBS medium likewise showed upregulated expressions of both genes compared to MT medium at day 7 (Figure 4B).

Expression profile analysis of cells revealed that the GAG-like scaffold instructs stem cells to differentiate into osteo/ chondrogenic lineages in concert with the FBS concentration present in the growth medium, such that a reduction in the FBS concentration acts as an inducer for osteogenic differentiation. GAG mimetic signals, when grafted on peptide nanofibers, may therefore serve as insoluble ligands that are organized into a nanoscale spatial matrix and enable the differentiation of stem cells, while the FBS amount controls the direction toward which the cells are inclined to differentiate.

Overall, the investigation of stem cell differentiation into osteogenic and chondrogenic lineages in 3D scaffolds showed that peptide nanofiber gel niches may exhibit varying effects on the direction of lineage commitment. However, in addition to the differential effects of PA gels and culture conditions, the heterogenic features of mesenchymal stem cells may also contribute to the differentiation process. As such, the immunophenotypic features of the cultured cell population were also investigated to better understand the natural and induced variances in the differentiation capacities of stem cells.

Immunophenotypic Characterization of Stem Cell Differentiation into Osteogenic and Chondrogenic Lineages. Analyzing the protein expression profile of mesenchymal stem cells is crucial for understanding the processes underlying the mechanism of their differentiation. However, one of the major problems of $3 \mathrm{D}$ cell culturing is the 
A
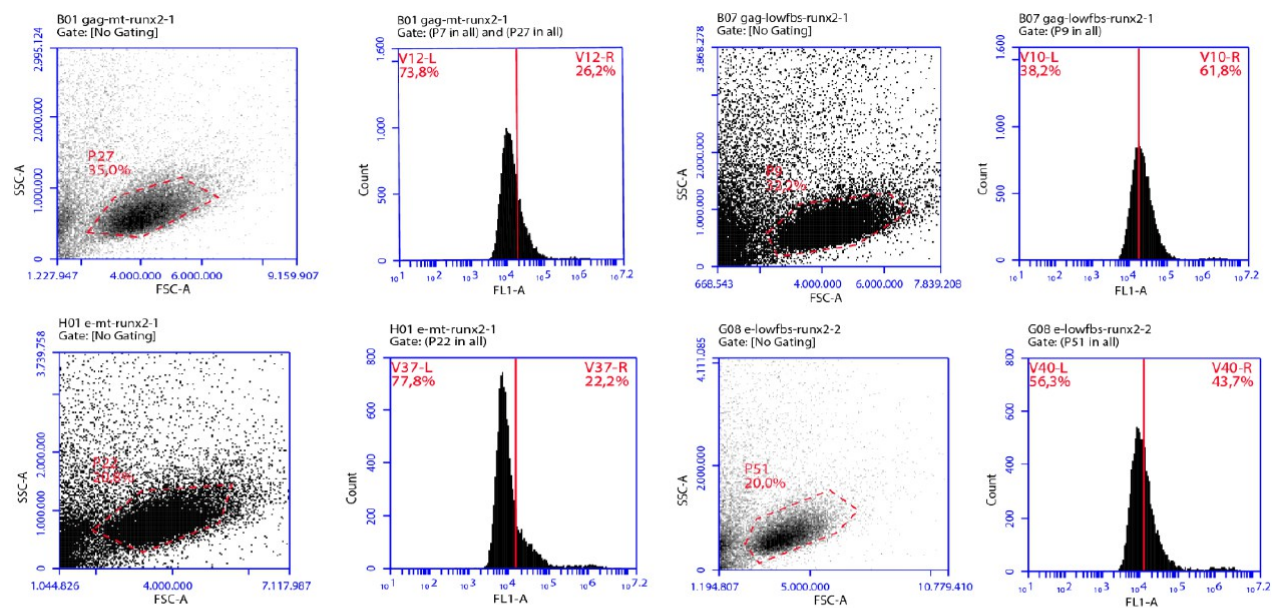

B

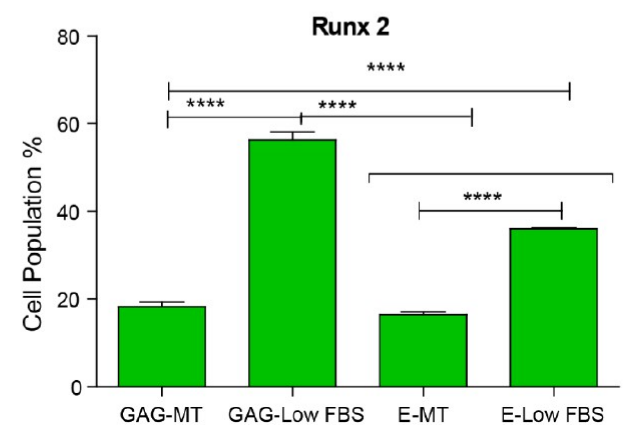

Figure 5. Immunophenotypic characterization of stem cells encapsulated in GAG- and E-gel niches for Runx2 expression on day 7. (A) Cell population and fluorescence signaling including corresponding gating are shown for both Runx2 labeling cells in GAG and E gel niches. Gating is applied for subtracting isotype labeling from Runx2 labeled populations, and the percent of remaining part is considered specifically stained with Runx2 antibody. (B) Cell population percentage comparison for Runx2 expression.

limitation of cellular and molecular analysis; for example, immunostaining of cells in a $3 \mathrm{D}$ microenvironment is highly tricky and requires advanced imaging technology for quantification. In this study, we optimized a flow cytometer protocol for the immunophenotypic characterization of stem cells during their differentiation. Therefore, we were able to analyze the protein production level and quantify cellular populations to examine the lineage commitment of a cell population at the single-cell level. Since cellular differentiation patterns were previously observed to be evident in as early as the seventh day of culture, flow cytometer analyses were performed at day 7 for the immunophenotypic characterization of stem cells cultivated on PA gels in MT medium or low-FBS medium.

Sox 9 and Runx 2 antibodies were used to quantify cells as either chondro-committed or osteo-committed, respectively, and the percentile distribution of the cell population was calculated for each experimental group (Figure 5 and Figure 6). Cells exposed to a combination of GAG-gel and MT medium were found to favor chondrogenesis instead of osteogenesis, exhibiting much higher Sox9 expression than Runx2. For other groups (GAG-gel/low FBS, E-gel/MT, E-gel/low FBS), however, cells with upregulated Runx2 expression were more numerous, indicating a predominance of osteogenic commitment. The concentration of serum also had a substantial effect on the differentiation response, as both GAG-MT and E-MT combinations favored chondrogenesis to a greater extent than their low-FBS counterparts.

Figures 5 and 6 show the osteo- and chondrogenic protein expression of cells, as investigated by immunophenotypic analysis for Runx 2 and Sox9. It is readily evident that the culture medium and the GAG-like 3D scaffold have separate and synergistic effects on the induction of stem cell differentiation. In MT medium, the GAG-like scaffold induced the upregulation of Sox 9 expression. Therefore, in the normal growth medium of stem cells, chemical and physical signatures presented in the GAG-gel niche are more likely to induce cells into chondrogenic differentiation. However, a lower concentration of FBS switches the effect of GAG-gel presence from pro-chondrogenic to pro-osteogenic. Even though low FBS media has pro-osteogenic effect in E-gel, it is notable that the effect of the MT media is considerably less prominent in the EPA gel niche; i.e., a similar number of cells undergo osteogenic and chondrogenic differentiation in E-MT. Although mRNA and protein expression levels of Runx2 correlate with each other, the mRNA and protein expression levels of Sox9 did not (Figure 4A and 6). The relative mRNA expression of Sox9 is lower than 1 or around 1, which shows that Sox9 expression was not significantly higher than the expression level of housekeeping gene $(\mathrm{GapdH})$. On the other hand, protein expression of Sox9 was significantly different among sample groups. Transcription and translation do not have linear 
A
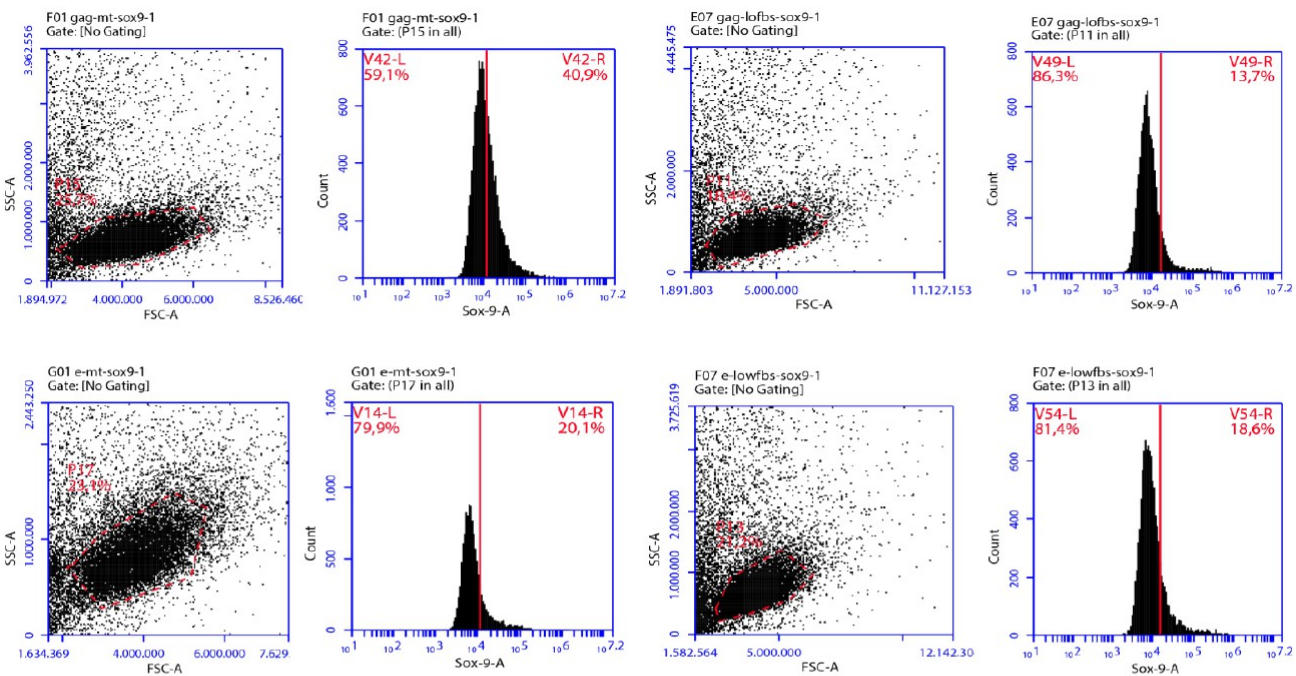

B

Sox-9

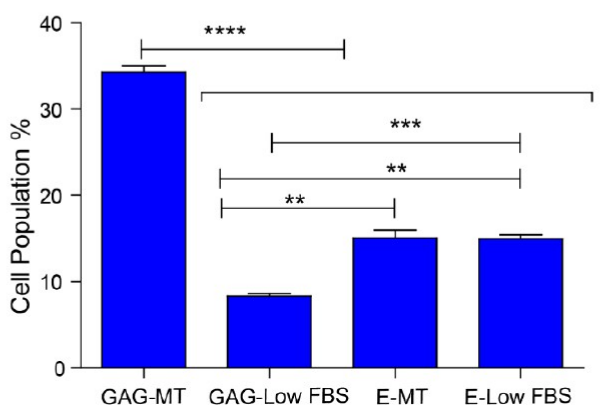

Figure 6. Immunophenotypic characterization of stem cells encapsulated in GAG- and E-gel niches for Sox9 expression on day 7. (A) Cell population and fluorescence signaling including corresponding gating is shown for Sox9 labeling cells in GAG and E gel niches. Gating is applied for subtracting isotype labeling from Sox 9 labeled populations, and percent of remaining part is considered specifically stained with Sox9 antibody. (B) Cell population percentage comparison for Sox 9 expression.

relationship; therefore, different regulatory mechanisms such as cis-acting and trans-acting mechanisms effect enhancement or repression of protein synthesis, which results in synthesis of proteins from a defined copy number of mRNA. ${ }^{33}$ Hence, we concluded that early activation of Sox 9 mRNA expression most probably occurs at initial days which leads to synthesis of Sox9 transcription factor in following days, and we observed increased protein synthesis at day 7 .

It is well-recognized that stem cells lose their differentiation potential outside of their natural niche. ${ }^{23}$ When designing artificial systems for the enhancement of tissue regeneration, various criteria should therefore be considered to ensure that cells are exposed to a faithful replication of their native environment. In our work, we showed that the differentiation potential of stem cells could be modulated by controlling the synergistic effects of a GAG-mimetic scaffold and the cell medium. However, in addition to osteogenic and chondrogenic lineages, mesenchymal stem cells exhibit the potential to differentiate into adipogenic and some other cell types. ${ }^{34}$ In addition, they are capable of self-renewal, which enables to the rapid proliferation of stem cells for tissue repair, but by necessity requires a population of undifferentiated cells. ${ }^{35}$ Our Sox 9 and Runx 2 expression results show that, in addition to osteo- and chondro-committed cells, a part of the population express neither of these markers and may be undifferentiated. Although these undifferentiated cells are generally underestimated and limit the utility of stem cells in regenerative medicine, we were able to decrease the percentage of nondifferentiating cells in osteo- and chondro-committed niches in GAG-low FBS and GAG-MT niches, suggesting that the GAG network also promotes differentiation in general.

These results overall indicate that GAG mimetic peptide nanofibers in the 3D PA gels induce osteogenic differentiation in the presence of low FBS media, while chondrogenic differentiation dominates when FBS levels are increased. Indeed, chondrogenic differentiation is only prominent when cells are grown in GAG-gel scaffolds and treated with MT medium, while cells in other groups are largely predisposed toward osteogenesis. As such, GAG-mimetic bioactive signals are able to initiate chondrogenesis in a much greater population of cells in MT medium. A similar trend is also observed in the nonbioactive gel niche, although to a lesser extent. We therefore conclude that MT medium alone is not able to support a dominant chondrogenic profile and that synergistic effects between the GAG-mimetic scaffold and the high-FBS 
medium is necessary for the majority of the cell population to commit to a chondrogenic differentiation pathway.

\section{CONCLUSION}

In this study, we investigated the potential of stem cells to differentiate into either osteogenic or chondrogenic lineages in 3D GAG-mimetic PA gel niches. GAG-like scaffolds were demonstrated to exhibit a distinctive effect on the differentiation of mesenchymal stem cells into osteo/chondrogenic lineages in media containing high and low concentrations of FBS. As mesenchymal stem cells are commonly used for the regeneration of cartilage and bone defects, characterization of their differentiation process is a topic of great interest for tissue engineering. We showed that 3D GAG-mimetic peptide nanofiber network can orchestrate the differentiation of mesenchymal stem cells in conjunction with environmental stimuli. It is noteworthy that differentiation potentials of stem cells could be regulated by altering medium components with the integrative effect of GAG mimetic scaffold. In addition, the 3D GAG gel was shown to be efficient for expanding of mesenchymal stem cells ex vivo, and the material can be developed further for in vivo applications. By combining this versatile GAG mimetic scaffold with optimum cell culture conditions, it is possible to direct the differentiation of stem cells into specific lineages with high efficiency. After the initial induction of cells into preosteogenic or prechondrogenic stages, the committed cells in these gels can also be selected (e.g., by using florescence activated cell sorting-FACS) for further clinical applications.

\section{ASSOCIATED CONTENT}

\section{S Supporting Information}

The Supporting Information is available free of charge on the ACS Publications website at DOI: 10.1021/acs.biomac.5b01637.

PA gel compositions, LC-MS results of PAs, STEM images of PA nanofibers, additional rheological characterizations of the PA gels and gel niches, Live/Dead assay results for PA gels and TCP, primer sequences for qRTPCR experiments, DMMB assay result for the quantification of sulfated glycosaminoglycan in gel niches, additional flow cytometry analyses, and zeta measurement analysis (PDF)

\section{AUTHOR INFORMATION}

\section{Corresponding Authors}

*E-mail address: moguler@unam.bilkent.edu.tr (M.O.G.).

*E-mail address: atekinay@bilkent.edu.tr (A.B.T).

\section{Author Contributions}

E.A., M.O.G., and A.B.T. designed the experiments, E.A. performed the experiments. The manuscript was written by all authors. All authors have given their approval to the final version of the manuscript.

\section{Notes}

The authors declare no competing financial interest.

\section{ACKNOWLEDGMENTS}

E.A. acknowledges support from TUBITAK-BIDEB fellowship. We would like to express our gratitude to $M$. Guler for assistance on TEM, to G. Gulseren for assistance on flow cytometry analysis, and to A. D. Ozkan for valuable comments on the manuscript. We thank TUBITAK grant number $111 \mathrm{M} 710$ for financial support. A.B.T. and M.O.G. acknowledge support from the Turkish Academy of Sciences Distinguished Young Scientist Award (TUBA-GEBIP).

\section{ABBREVIATIONS}

GAG, Glycosaminoglycan; 3D, three-dimensional; ECM, extracellular matrix; FBS, fetal bovine serum; PA, peptide amphiphile; CD, circular dichroism; STEM, sacking transmission electron microscopy; SEM, scanning electron microscopy; qRT-PCR, quantitative real time polymerase chain reaction; prep-HPLC, preparative high performance liquid chromatography; DMMB, dimethylmethylene blue

\section{REFERENCES}

(1) Frantz, C.; Stewart, K. M.; Weaver, V. M. J. Cell Sci. 2010, 123, 4195-4200.

(2) Giuliani, N.; Lisignoli, G.; Magnani, M.; Racano, C.; Bolzoni, M.; Dalla Palma, B.; Spolzino, A.; Manferdini, C.; Abati, C.; Toscani, D. Stem Cells Int. 2013, 2013, 312501.

(3) Kang, S. K.; Shin, I. S.; Ko, M. S.; Jo, J. Y.; Ra, J. C. Stem Cells Int. 2012, 2012, 342968.

(4) Arslan, E.; Garip, I. C.; Gulseren, G.; Tekinay, A. B.; Guler, M. O. Adv. Healthcare Mater. 2014, 3, 1357-1376.

(5) Baker, B. M.; Chen, C. S. J. Cell Sci. 2012, 125, 3015-3024.

(6) Alberts, B.; Johnson, A.; Lewis, J. et al. Molecular Biology of the Cell, 4th ed.; Garland Science: New York, 2002.

(7) Gao, Y.; Liu, S.; Huang, J.; Guo, W.; Chen, J.; Zhang, L.; Zhao, B.; Peng, J.; Wang, A.; Wang, Y.; Xu, W.; Lu, S.; Yuan, M.; Guo, Q. BioMed Res. Int. 2014, 2014, 648459.

(8) Kyllönen, L.; Haimi, S.; Mannerström, B.; Huhtala, H.; Rajala, K. M.; Skottman, H.; Sándor, G. K.; Miettinen, S. Stem Cell Res. Ther. 2013, 4,17

(9) Mackay, A. M.; Beck, S. C.; Murphy, J. M.; Barry, F. P.; Chichester, C. O.; Pittenger, M. F. Tissue Eng. 1998, 4, 415-428.

(10) Niece, K. L.; Hartgerink, J. D.; Donners, J. J. J. M.; Stupp, S. I. J. Am. Chem. Soc. 2003, 125, 7146-7147.

(11) Behanna, H. A.; Donners, J. J. J. M.; Gordon, A. C.; Stupp, S. I. J. Am. Chem. Soc. 2005, 127, 1193-1200.

(12) Pierschbacher, M. D.; Ruoslahti, E. Nature 1984, 309, 30-33.

(13) Hersel, U.; Dahmen, C.; Kessler, H. Biomaterials 2003, 24 (24), 4385-4415.

(14) Fox, A. J. S.; Bedi, A.; Rodeo, S. A. Sports Health 2009, 1, 461468

(15) Shah, R. N.; Shah, N. A.; Del Rosario Lim, M. M.; Hsieh, C.; Nuber, G.; Stupp, S. I. Proc. Natl. Acad. Sci. U. S. A. 2010, 107, 32933298.

(16) Lodish, H.; Berk, A.; Zipursky, S. L.; Matsudaira, P.; Baltimore, D.; Darnell, J. Molecular Cell Biology, 4th ed.; Freeman, W. H.: New York, 2000.

(17) Lee, C. H.; Singla, A.; Lee, Y. Int. J. Pharm. 2001, 221, 1-22.

(18) Spinelli, F. J.; Kiick, K. L.; Furst, E. M. Biomaterials 2008, 29, 1299-1306.

(19) Lin, S.; Gu, L. Materials 2015, 8, 551-560.

(20) Burdick, J. A.; Prestwich, G. D. Adv. Mater. 2011, 23, H41-H56.

(21) Toksoz, S.; Mammadov, R.; Tekinay, A. B.; Guler, M. O. J. Colloid Interface Sci. 2011, 356, 131-137.

(22) Gaspar, D. A.; Gomide, V.; Monteiro, F. J. Biomatter 2012, 2, $167-175$.

(23) Lutolf, M.; Hubbell, J. Nat. Biotechnol. 2005, 23, 47-55.

(24) Hosseinkhani, H.; Hong, P.-D.; Yu, D.-S. Chem. Rev. 2013, 113, $4837-4861$.

(25) Zein, I.; Hutmacher, D. W.; Tan, K. C.; Teoh, S. H. Biomaterials 2002, 23, 1169-1185.

(26) Lin, A. S.; Barrows, T. H.; Cartmell, S. H.; Guldberg, R. E. Biomaterials 2003, 24, 481-489. 
(27) Oxley, H.; Corkhill, P.; Fitton, J.; Tighe, B. Biomaterials 1993, $14,1064-1072$.

(28) Chirila, T. V.; Constable, I. J.; Crawford, G. J.; Vijayasekaran, S.; Thompson, D. E.; Chen, Y.-C.; Fletcher, W. A.; Griffin, B. J. Biomaterials 1993, 14, 26-38.

(29) Chen, G.; Ushida, T.; Tateishi, T. Mater. Sci. Eng., C 2001, 17, 63-69.

(30) Taboas, J.; Maddox, R.; Krebsbach, P.; Hollister, S. Biomaterials 2003, 24, 181-194.

(31) Hosseinkhani, H.; Hong, P.-D.; Yu, D.-S.; Chen, Y.-R.; Ickowicz, D.; Farber, I.-Y.; Domb, A. J. Int. J. Nanomed. 2012, 7, 3035.

(32) Haines-Butterick, L.; Rajagopal, K.; Branco, M.; Salick, D.; Rughani, R.; Pilarz, M.; Lamm, M. S.; Pochan, D. J.; Schneider, J. P. Proc. Natl. Acad. Sci. U. S. A. 2007, 104, 7791-7796.

(33) Maier, T.; Güell, M.; Serrano, L. FEBS Lett. 2009, 583, 39663973.

(34) Kalervo Väänänen, H. Ann. Med. 2005, 37, 469-479.

(35) Kumar, S.; Chanda, D.; Ponnazhagan, S. Gene Ther. 2008, 15, 711-715. 